looking through distraction cards/Flippits ${ }^{\circledR}$ and kaleidoscope to reduce procedural pain during blood draw in children between the ages of 7-11. Our secondary aim is to evaluate venipuncture success during the procedure.

Methods This is a prospective randomized clinical trial. The study was conducted at the phlebotomy station of Karaman Maternity and Children Hospital, Karaman Turkey. Inclusion criteria were 7-11 year-old patients who required blood tests. The data was obtained by interviewing the children, their parents and the observer. Procedural pain levels of children was assessed by parent and observer reports using Visual Analog Scale (VAS) and Wong Baker pain scale. Data that were analyzed with SPSS 15.00. $\mathrm{p}<0.05$ was considered as significant. Parametric data such as pain level of children were compared with the $t$ test. Nonparametric data such as gender and success of blood drawing procedure were compared with percentage of frequency and $x^{2}$ comparisons.

Results Both groups have significantly lower pain levels than the control group $(\mathrm{p}<0.05)$

Conclusion The result of the study suggests that the both distraction methods effectively decreased pain and anxiety levels of children compared to control group according to self-reports, parent' and observer' reports.

\section{THE RELATION BETWEEN HOSPITAL DESIGN AND HOSPITAL INFECTION}

doi:10.1136/archdischild-2012-302724.0078

S Youssef. Infection Control, Faculty of Pharmacy, Org. of Teaching Hospitals Institutes, Alexandria, Egypt

Objectives The present study was done at Damanhour Teaching Hospital to identify the following aims:

To estimate the prevalence rate \% of NI among different dept. in the hospital.

To determine the influence of the number of sinks on the NI rate $\%$. To assess the knowledge of the medical staff regarding the importance and the proper way of hand washing.

To focus on the importance of hospital design in developing countries.

Patients and Methods The target population for this study consisted of 217 patients admitted at the hospital $\varsigma<=48$ hours. A questionnaire was developed by the researchers after reviewing literature. This questionnaire composed of two parts, the first part elicited the clinical examination supported by the laboratory and $x$-rays investigations to assure the exactly number of the nosocomial infected patients among 217 patients the, second part included questions covering the number of sinks, number of occupied beds, number of medical staff in the chosen hospital departments. Samples were taken from the wounds aerobically and nonaerobically.

Results and discussion The sinks are completely insufficient for hand washing (about one sink for every 40 medical staff). The data showed that E.coli is the microorganism most commonly isolated from nosocomial infected patients which improve the improper hand washing and the poor personal hygiene.

\section{RACIAL/ETHNIC DISPARITIES IN CENTER FOR DISEASE CONTROL AND PREVENTION (CDC) PEDIATRIC VACCINATION SCHEDULE AND RECOMMENDATION IN UNITED STATES}

doi:10.1136/archdischild-2012-302724.0079
1,2,3 L Holmes, 'P Oceanic, 'D Fitzgerald, 'K Grant, 1,2K Dabney. ${ }^{1}$ Health Equity Plus Inclusion; ${ }^{2}$ Orthopedic Department, Nemours/A.I. Dupont Children Hospital, Wilmington; ${ }^{3}$ Biological Sciences, University of Delaware, Newark, DE, USA

Background and aim Children require age-specific vaccination in order to prevent against childhood morbidities and mortality. The CDC provides the guidelines for immunization schedule. This study aimed to assess the prevalence of age-specific vaccination schedule, and to determine whether or not Asians demonstrate better compliance relative to other racial/ethnic groups.

Methods We retrospectively assessed a prospectively collected data on vaccination received in our institution during 2010. To test the study specific hypothesis on racial/ethnic disparities in compliance with the CDC recommendation, we used chi squared and multivariable logistic regression model.

Results There were 5867 children who received vaccines during this period. The racial distribution indicated: Whites/Caucasians, 1,917(32.7\%), Blacks/African Americans (AA), 2904(49.5\%), Asian, 134(2.3\%), Hawaiian native/Pacific Islander,4 (0.1\%), American Indian/Alaskan Native(AI/AN), $9(0.2 \%)$, and some other race, $727(12.4 \%)$. Asians (97.0\%) relative to AA (93.1\%) and Caucasian (91\%) demonstrated the highest compliance in the age-specific vaccines combined, $\chi^{2}(7)=24.5, p=0.001$. With crude analysis, AA and Caucasians, relative to Asians were $58 \%$ (Odds ratio $[\mathrm{OR}]=0.42$, 95\% CI, 0.15-1.14), and 69\%, (OR=0.31, 95\%CI, 0.11-0.85) less likely to adhere to the CDC schedule respectively. However, after controlling for insurance status, the significant racial disparities did not persist between Asians and Caucasians, adjusted OR, 0.45, 99\%CI, 0.08-1.11.

Conclusion In a large pediatric cohort, Asians demonstrated highest compliance in vaccine schedule, indicative of racial disparities. Therefore, knowledge of predisposing factors to impaired compliance among racial/ethnic groups in vaccines schedule may assist in narrowing health disparities in this direction as well as facilitate our efforts in addressing preventable childhood diseases.

\section{MOBILE CARDIORESPIRATORY EVENT MONITORING FOR VACCINATION IN FORMER EXTREMELY PRETERM INFANTS}

doi:10.1136/archdischild-2012-302724.0080

${ }^{1} \mathrm{FF}$ Pulzer, ${ }^{2} \mathrm{M}$ Quante, ${ }^{2} \mathrm{~J}$ Kluge, ${ }^{1} \mathrm{C}$ Gebauer, ${ }^{1} \mathrm{M}$ Knüpfer, ${ }^{1} \mathrm{U}$ Thome. ${ }^{1}$ Neonatology: 2Pediatrics, University Children's Hospital, Leipzig, Germany

Background and aims Recommendations concerning the assessment of cardiorespiratory events during the first immunization with diphtheria-tetanus-pertussis-inactivated polio-Haemophilus influenzae type B (DTP-IPV-Hib) and Pneumococcal conjugate vaccine (PCV) of extremely preterm infants are discussed controversely. We examined the relationship between the immunization and cardiorespiratory events in preterm infants by using a mobile event monitor.

Methods We enrolled 84 extremely preterm infants [39 girls, 45 boys; gestational age (GA) < 28.0 weeks (range 23.5-27.6)]. Immunization took place in the last week before discharge (mean GA: 38 weeks). Recording monitors were used continuously 12 hours before and during 48 hours after immunization to document prolonged apnea and bradycardia.

Results The incidence of adverse cardiorespiratory events postimmunization (PI) was higher in the whole group with $40 \%$ of the infants having apneas $>3$ seconds longer than before immunization (BI), and more prolonged events of bradycardia. The longest apnea observed PI was 20 seconds. Mean PI desaturations were more pronounced (76\% PI vs. $67 \%$ BI; $p<0.05)$. Furthermore, during the first 24 hours PI the mean oxygen saturation was lower, and the mean heart rate was significantly higher. In $40 \%$ of the children the second 
immunization was performed under continuous cardiorespiratory monitoring.

Conclusions Preterm infants who received diphtheria-tetanuspertussis-inactivated polio-Haemophilus influenzae type B and pneumococcal vaccine before discharge were more likely to temporarily experience prolonged apnea and bradycardia after immunization. Continuous mobile event monitoring of these infants was a helpful tool to detect clinically significant cardiorespiratory events.

\section{EASY FOR YOU TO SAY! ADHERENCE ISSUES IN ADOLESCENCE}

doi:10.1136/archdischild-2012-302724.0081

JC Suris. Department of Pediatrics, Lausanne University Hospital, Lausanne, Switzerland

Adherence to treatment (defined as the active, voluntary, collaborative involvement of the patient in a mutually acceptable course of behavior to produce a desired preventive or therapeutic result) is not always simple, and for adolescents suffering from chronic conditions even less.

Adolescence is a time of transitions: from childhood to adulthood, from education to employment, from pediatrics to adult health, from controlled treatment to treatment control. This means that they have to manage their treatment (which may cause problems with their parents who had this function until then) and have the right to disagree (which may cause problems with their health care providers).

In fact, among adolescents with chronic diseases, treatment adherence rates vary widely depending on the condition: from $98-99 \%$ for cancer to $77 \%$ for mild asthma or $55-86 \%$ for renal transplant recipients, for example. Nevertheless, and although selfreport is often overestimated, part of the variation among adherence rates is also due to what kind of cut-off point for good/ acceptable adherence is used.

Although there are many factors influencing adherence (such as side effects, taste, motivation or severity of the condition to mention a few), there is fair amount of literature related to the predictors of a good or a poor adherence. This presentation will explore the main causes of non-adherence among youths and practical and relatively simple ways to increase their adherence rate.

\section{STABILITY OF LUNG FUNCTION TESTS BETWEEN 8 AND 18 YEARS OF AGE OF EPT/ELBW SURVIVORS COMPARED WITH TERM CONTROLS}

doi:10.1136/archdischild-2012-302724.0082

1.2.3LW Doyle, ${ }^{4} \mathrm{~A}$ Gibson, ${ }^{\mathrm{J}}$ Cheong, ${ }^{4} \mathrm{G}$ Roberts, ${ }^{4} \mathrm{C}$ Robertson, The Victorian Infant Collaborative Study Group. 'Royal Women's Hospital; ' ${ }^{2}$ nniversity of Melbourne; ${ }^{3}$ Murdoch Children's Research Institute; ${ }^{4}$ Royal Childrens Hospital Melbourne, Parkville, VIC, Australia

Aims To determine the stability of lung function test results of extremely preterm (EPT; $<28$ weeks' gestational age) or extremely low birthweight (ELBW; birthweight $<1000 \mathrm{~g}$ ) survivors between 8 and 18 years of age compared with term controls.

Methods Eighteen-year follow-up of participants born either EPT/ELBW in 1991-92 in the state of Victoria and randomly selected term, normal birthweight controls. Lung volumes and flows, including the forced expired volume in 1 second $\left(\mathrm{FEV}_{1}\right)$, forced vital capacity (FVC), the $\mathrm{FEV}_{1} / \mathrm{FVC}$, and the forced expiratory flow $\left(\mathrm{FEF}_{25-75 \%}\right)$ were measured at 8 and 18 years of age according to standard guidelines and results converted to Z-scores for age, height and gender.
Results Lung function data were obtained from 187/298 (63\%) EPT/ELBW subjects and 147/262 (56\%) controls at both 8 and 18 years. There were strong positive relationships between lung function results at 18 years with results obtained at 8 years in both groups (Table).

\section{Abstract 82 Table 1}

\begin{tabular}{lcccc}
\hline Lung function variable & EPT/ELBW & \% variance & Controls & \% variance \\
\hline FEV1 & $0.887(0.058)^{*}$ & $56.0 \%$ & $0.554(0.071)^{*}$ & $29.4 \%$ \\
FVC & $0.676(0.058)^{*}$ & $42.2 \%$ & $0.586(0.067)^{*}$ & $34.5 \%$ \\
FEV1/FVC & $0.422(0.051)^{*}$ & $26.8 \%$ & $0.384(0.063)^{*}$ & $20.5 \%$ \\
FEF25\%-75\% & $0.772(0.057)^{*}$ & $50.3 \%$ & $0.561(0.070)^{*}$ & $30.7 \%$ \\
\hline
\end{tabular}

Changes in Respiratory Variables Between 8 and 18

Data are regression coefficients $(\mathrm{SE}) ;{ }^{*} P<0.001$

Relationships between 8 and 18 years were significantly stronger for EPT/ELBW subjects for both $\mathrm{FEV}_{1}$ and $\mathrm{FEF}_{25-75 \%}$ compared with controls.

Conclusions There are strong linear relationships between lung function values at 8 and 18 years in both EPT/ELBW survivors and controls.

\section{LEVEL OF SELF-SUFFICIENCY OF YOUNG PEOPLE WITH DOWN SYNDROME (AGE 16-19 YEARS OLD)}

doi:10.1136/archdischild-2012-302724.0083

M Fekkes, H van Gameren-Oosterom, J van Wouwe. TNO Child Health, TNO, Leiden Belgium

Background and aims Becoming independent is for young people with Down syndrome (DS) not a standard development, because of their intellectual impairment. Parents often wonder what level their child with DS might reach. This study aims to measure the level of self-sufficiency and problem behaviour in a large population based sample of 16-19-year-old people with DS

Methods Parents of Dutch children with DS born in 1992, 1993 and 1994 were invited to participate $(n=513)$. Data were collected by a written questionnaire for the parents, containing the Dutch SRZ (measuring social independence), the Child Behaviour Checklist, the Children's Social Behaviour Questionnaire, and additional questions on skills and background. Differences between mean values were evaluated using t-tests.

Results Mean age of the 322 participating young people with DS was 18.3 years $(52.8 \%$ boys). Considering their basic daily skills, they reached a level of around $70 \%$ of independent functioning. Girls had a higher level of functioning than boys ( $<<0.001)$, except for the subscale social orientation. With regards to behaviour problems the young people with DS had more behavioural problems on the subscales social problems, thought problems and attention problems. Only on the subscale anxious/depressed young people with DS scored fewer problems $(p<0.001)$. Social problems were most present in the subscales orientation problems, not understanding social situations, and resistance to changes.

Conclusions Young people with DS do not reach a complete level of independent functioning. Behavioural and social problems have an important contribution to the limited self-sufficiency of this group.

\section{HOW CAN WE IMPROVE TISSUE PERFUSION AFTER CARDIAC SURGERY}

doi:10.1136/archdischild-2012-302724.0084

E da Cruz. Pediatrics, Children's Hospital Colorado, University of Colorado Denver, School of Medicine, Aurora, CO, USA 